\title{
Iterative Refinement Methods for Time-Domain Equalizer Design
}

\author{
Güner Arslan, ${ }^{1}$ Biao Lu, ${ }^{2}$ Lloyd D. Clark, ${ }^{3,4}$ and Brian L. Evans ${ }^{5}$ \\ ${ }^{1}$ Silicon Laboratories, Corporate Headquarters, 7000 West William Cannon Drive, Austin, TX 78735, USA \\ ${ }^{2}$ Schlumberger Sugar Land Product Center, 110 Schlumberger Drive, Sugar Land, TX 77478, USA \\ ${ }^{3}$ Schlumberger Austin Systems Center, 8311 N FM 620 Road, Austin, TX 78726, USA \\ ${ }^{4}$ TICOM Geomatics, 9130 Jollyville Road, Austin, TX 78759, USA \\ ${ }^{5}$ Department of Electrical and Computer Engineering, The University of Texas, Austin, TX 78712-1084, USA
}

Received 1 December 2004; Revised 23 May 2005; Accepted 2 August 2005

Commonly used time domain equalizer (TEQ) design methods have been recently unified as an optimization problem involving an objective function in the form of a Rayleigh quotient. The direct generalized eigenvalue solution relies on matrix decompositions. To reduce implementation complexity, we propose an iterative refinement approach in which the TEQ length starts at two taps and increases by one tap at each iteration. Each iteration involves matrix-vector multiplications and vector additions with $2 \times 2$ matrices and two-element vectors. At each iteration, the optimization of the objective function either improves or the approach terminates. The iterative refinement approach provides a range of communication performance versus implementation complexity tradeoffs for any TEQ method that fits the Rayleigh quotient framework. We apply the proposed approach to three such TEQ design methods: maximum shortening signal-to-noise ratio, minimum intersymbol interference, and minimum delay spread.

Copyright (C) 2006 Güner Arslan et al. This is an open access article distributed under the Creative Commons Attribution License, which permits unrestricted use, distribution, and reproduction in any medium, provided the original work is properly cited.

\section{INTRODUCTION}

Multicarrier modulation is a widely used modulation method for reliable high-speed communication. Discrete multitone (DMT) modulation is a popular variant of multicarrier modulation that has been standardized for asymmetric and very high-speed digital subscriber loops (ADSL and VDSL, resp.) [1]. In these applications, a guard sequence known as the cyclic prefix is prepended to each symbol to help the receiver eliminate intersymbol interference (ISI) and perform symbol recovery.

A DMT symbol consists of $N$ samples, and the cyclic prefix is a copy of the last $v$ samples of the symbol. The length of the channel impulse response has to be less than or equal to $(v+1)$ samples in order for all ISI to be eliminated. Using a cyclic prefix, however, reduces the channel throughput of a DMT transceiver by a factor of $\nu /(N+\nu)$. Therefore, it is desirable to choose $v$ as small as possible.

The ADSL and VDSL standards set $v$ to be N/16. In the field, however, ADSL and VDSL channel impulse responses can exceed N/16 samples. It is up to the equalizer in the receiver to shorten the channel impulse response and to correct for frequency distortion in the shortened channel. These two equalization tasks may be decoupled or combined
[2]. In a decoupled approach, the equalizer is a cascade of a time-domain equalizer (TEQ) to shorten the channel, a fast Fourier transform (FFT) to perform multicarrier demodulation, and a frequency-domain equalizer (FEQ) to invert the frequency response of the shortened channel [3]. These three operations are linear. Combined equalization approaches exploit the linearity by either moving the TEQ into the FEQ to yield per-tone equalizers [4], or moving the FEQ into the TEQ to yield complex-valued time-domain equalizer filter banks [5]. Combined equalization approaches yield higher data rates than decoupled approaches for the downstream ADSL case [2].

A TEQ is generally implemented as a finite impulse response (FIR) filter placed at the receiver. The cascade of the channel impulse response and the TEQ forms an effective channel impulse response with length of $\nu+1$ samples, as shown in Figure 1. (In the case of ADSL, the channel impulse response is actually shortened to $v$ samples.) Various design criteria resulting in many different design methods have been proposed to calculate the TEQ coefficients [3, 68]. These four cited design methods can be unified as an optimization problem involving a Rayleigh quotient [2]. The generalized eigenvalue solution using matrix decompositions 


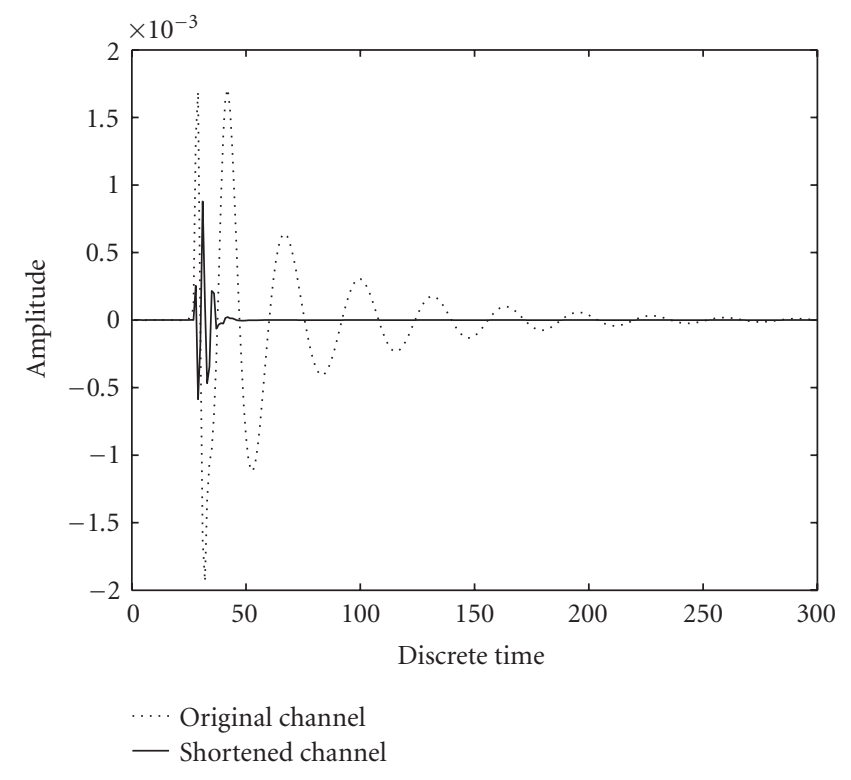

Figure 1: Example of the channel impulse response (carrier serving area loop 1), and the shortened channel impulse response obtained with a 16-tap TEQ designed with a maximum shortening signal-tonoise ratio (MSSNR) method.

is in general not practical to implement in real-time on programmable digital signal processors.

Instead, iterative design methods could be applied. The iterative method could fix the TEQ length, $N_{w}$, and use gradient descent based on the Rayleigh quotient formulation to iterate towards an optimal answer [9]. The step size must be chosen with care, and scaling (normalization) may be needed at each iteration. Although each iteration depends on matrix-vector multiplications and vector additions involving $N_{w} \times N_{w}$ matrices and vectors of length $N_{w}$, matrix decompositions are avoided.

We propose an iterative refinement approach in which the TEQ length starts at two taps and increases by one tap at each iteration. A maximum TEQ length may be set. Other stopping criteria include the cases in which no significant improvement in the objective function over the previous iteration, and cases in which the objective function value has degraded over the previous iteration. Hence, the approach will improve the design at each iteration until it terminates. No step size needs to be chosen and no scaling is needed. Each iteration involves matrix-vector multiplications and vector additions but involving $2 \times 2$ matrices and two-element vectors. Provided that the proposed approach completes its initialization step, the proposed approach can be terminated at any time and a useful TEQ will result. Hence, our approach scales with the available computational resources.

We apply the iterative refinement approach to the objective functions of three different TEQ design methods: maximum shortening signal-to-noise ratio (MSSNR) [6], minimum intersymbol interference (min-ISI) [7], and minimum delay spread (MDS) [8] methods. For each TEQ de- sign method, we develop two iterative refinement algorithms. The divide-and-conquer Rayleigh quotient (DC-Rayleigh) algorithm uses the objective function in Rayleigh quotient form. The divide-and-conquer eigenvector algorithm (DCeigenvector) optimizes the numerator of the objective function subject to a constraint involving the TEQ. The DCeigenvector algorithm will have lower implementation complexity than the DC-Rayleigh algorithm, which in turn will have significantly lower complexity than the originally reported TEQ design method.

The rest of the paper is organized as follows. Section 2 summarizes the three TEQ design methods of interest with their objective functions. Section 3 derives the closed-form solutions for the DC-Rayleigh and DC-eigenvector methods. Section 4 applies the DC-Rayleigh and DC-eigenvector methods to three TEQ design methods. Section 5 shows detailed simulation results for the proposed methods. Section 6 concludes the paper.

\section{BACKGROUND}

In this section, we summarize three existing TEQ design methods and the objective functions they optimize. All methods assume that $v$ is the length of the cyclic prefix, that the equalized or effective channel impulse response has a total delay of $\Delta$ samples, and that perfect knowledge of the channel impulse response is available. In ADSL and VDSL, the channel impulse response can be estimated during training. During training, the discrete Fourier transform (DFT) of the channel impulse response is estimated, from which we can obtain the channel impulse response estimate. The effect of channel estimation error on the following TEQ design methods has been quantified in [10].

\subsection{The maximum shortening signal-to-noise ratio method}

Melsa et al. [6] approach the TEQ design as solely a channel shortening problem. They define a shortening signal-tonoise (SSNR) and derive the optimal TEQ in terms of maximizing SSNR which is the ratio of the energy inside a window of $(\nu+1)$ samples starting at sample $(\Delta+1)$ to the energy outside the same window of the shortened channel impulse response. An ideal shortened channel impulse response would be zero-valued outside the window in order to yield zero ISI and infinite SSNR. The assumption is that the larger the SSNR, the closer the shortened channel impulse response is to the ideal. However, optimizing SSNR is not necessarily equivalent to maximizing bitrate or minimizing biterror rate but only an approximation to make the TEQ design problem mathematically tractable. Although this method ignores all noise components simulation results show that it performs comparably well to other methods that take noise into account [2].

Let us define the effective or shortened channel impulse response $h_{\text {eff }}(k)$ as

$$
h_{\mathrm{eff}}(k)=h(k) * w(k),
$$


where $h(k)$ is the channel impulse response of length $L_{h}$, $w(k)$ represents the $N_{w}$ TEQ coefficients, and “*” denotes linear convolution. We can represent $h_{\mathrm{eff}}(k)$ in vector form as

$$
\mathbf{h}_{\mathrm{eff}}=\left[h_{\mathrm{eff}}(1), h_{\mathrm{eff}}(2), \ldots, h_{\mathrm{eff}}\left(L_{h}+N_{w}-1\right)\right] .
$$

The goal is to choose the TEQ coefficients such that the energy of the effective channel impulse response $\mathbf{h}_{\text {eff }}$ mostly concentrates inside a window with length $v+1$, one sample longer than the cyclic prefix. To accomplish this goal, we split $\mathbf{h}_{\text {eff }}$ into two parts, $\mathbf{h}_{\text {win }}$ and $\mathbf{h}_{\text {wall }}$, which represent samples of the effective channel impulse response inside and outside the window [6], respectively:

$$
\begin{aligned}
& \mathbf{h}_{\text {win }} \\
& =\left[h_{\mathrm{eff}}(\Delta+1), h_{\mathrm{eff}}(\Delta+2), \ldots, h_{\mathrm{eff}}(\Delta+v+1)\right], \\
& \mathbf{h}_{\mathrm{wall}} \\
& =\left[h_{\mathrm{eff}}(1), \ldots, h_{\mathrm{eff}}(\Delta), h_{\mathrm{eff}}(\Delta+v+2), \ldots, h_{\mathrm{eff}}\left(L_{h}+N_{w}-1\right)\right] .
\end{aligned}
$$

The samples in $\mathbf{h}_{\text {wall }}$ include the samples before the window and the samples after the window. The SSNR objective function [6] is defined as

$$
\mathrm{SSNR}=10 \log _{10} \frac{\text { Energy in } \mathbf{h}_{\text {win }}}{\text { Energy in } \mathbf{h}_{\text {wall }}},
$$

where $\mathbf{h}_{\text {win }}$ and $\mathbf{h}_{\text {wall }}$ can be written in matrix form as shown in the following:

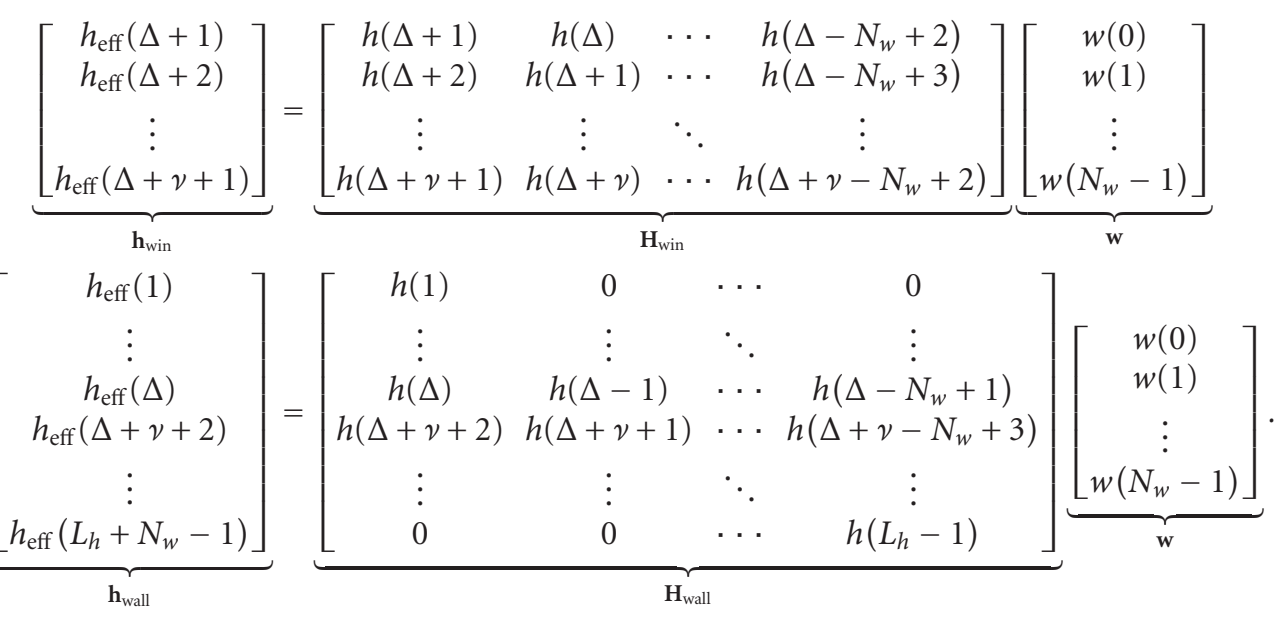

The energy of $\mathbf{h}_{\text {win }}$ and $\mathbf{h}_{\text {wall }}$ in (4) can be written as

$$
\begin{aligned}
\mathbf{h}_{\text {wall }}^{T} \mathbf{h}_{\text {wall }} & =\mathbf{w}^{T} \mathbf{H}_{\text {wall }}^{T} \mathbf{H}_{\text {wall }} \mathbf{w}=\mathbf{w}^{T} \mathbf{A} \mathbf{w}, \\
\mathbf{h}_{\text {win }}^{T} \mathbf{h}_{\text {win }} & =\mathbf{w}^{T} \mathbf{H}_{\text {win }}^{T} \mathbf{H}_{\text {win }} \mathbf{w}=\mathbf{w}^{T} \mathbf{B} \mathbf{w},
\end{aligned}
$$

where the $N_{w} \times N_{w}$ matrices are defined as

$$
\begin{aligned}
& \mathbf{A}=\mathbf{H}_{\text {wall }}^{T} \mathbf{H}_{\text {wall }} \\
& \mathbf{B}=\mathbf{H}_{\text {win }}^{T} \mathbf{H}_{\text {win }} .
\end{aligned}
$$

Note that both $\mathbf{A}$ and $\mathbf{B}$ are real, symmetric and positive definite (excluding the case of ideal equalization which is not possible in practice) by definition. SSNR can then be written in compact form as

$$
\mathrm{SSNR}=10 \log _{10} \frac{\mathbf{w}^{T} \mathbf{B} \mathbf{w}}{\mathbf{w}^{T} \mathbf{A w}} .
$$

This form is known as the Rayleigh quotient. The optimal shortening method would find $\mathbf{w}$ to minimize $\mathbf{w}^{T} \mathbf{A w}$ while satisfying $\mathbf{w}^{T} \mathbf{B w}=1[6]$. Solving this problem via the Lagrange multiplier method easily yields the solution that $\mathbf{w}$ should be chosen as the generalized eigenvector of $\mathbf{B}$ and $\mathbf{A}$ corresponding to the largest generalized eigenvalue [11].

The approach in [6] to find the solutions is based on the assumption that $\mathbf{B}$ is positive definite so that it has a Cholesky decomposition as, $\mathbf{B}=\sqrt{\mathbf{B}} \sqrt{\mathbf{B}}$. Then, $\mathbf{l}_{\text {min }}$ is computed as the eigenvector associated with the smallest eigenvalue of the matrix $(\sqrt{\mathbf{B}})^{-1} \mathbf{A}\left(\sqrt{\mathbf{B}^{T}}\right)^{-1}$. Finally, $\mathbf{w}_{\text {opt }}=\left(\sqrt{\mathbf{B}}^{T}\right)^{-1} \mathbf{l}_{\min }$.

A more complicated method in [6] applies when $\mathbf{B}$ is singular. In order to avoid $\mathbf{B}$ from being singular, Yin and Yue [12] suggest an objective function to maximize $\mathbf{w}^{T} \mathbf{B w}$ while satisfying the constraint $\mathbf{w}^{T} \mathbf{A w}=1$. In this case, they assume that $\mathbf{A}$ is positive definite since they perform a Cholesky decomposition on A. Both cases $[6,12]$ require a Cholesky decomposition, an eigendecomposition, and a matrix inversion of an $N_{w} \times N_{w}$ matrix to find $\mathbf{w}_{\text {opt }}$.

\subsection{The minimum intersymbol interference method}

Arslan et al. [7] propose a TEQ design method that can be viewed as a generalization to the MSSNR method. The minimum intersymbol interference ( $\mathrm{min}-\mathrm{ISI}$ ) method is also 
a simplified version of the maximum bitrate TEQ [7] design method that directly optimizes bitrate based on a subchannel SNR model:

$$
\mathrm{SNR}_{i}=\frac{S_{x, i}\left|C_{\text {signal }, i}\right|^{2}}{S_{n, i}\left|C_{\text {noise }, i}\right|^{2}+S_{x, i}\left|C_{\mathrm{ISI}, i}\right|^{2}},
$$

where $S_{x, i}, S_{n, i}, C_{\text {signal }, i}, C_{\text {noise }, i}$, and $C_{\text {ISI }, i}$ are the signal power, noise power, signal path gain, noise path gain, and ISI path gain in the $i$ th subchannel, respectively. The min-ISI method makes use of the observation that the ISI term in the subchannel SNR model is the dominant factor limiting bitrate; hence, minimizing ISI alone would be a viable alternative to the Maximum Bit Rate (MBR) method [7] that otherwise requires nonlinear optimization to calculate the TEQ taps. The objective function for the min-ISI method can also be written as a Rayleigh quotient with matrices $\mathbf{A}$ and $\mathbf{B}$ defined as:

$$
\begin{gathered}
\mathbf{A}=\mathbf{H}^{T} \mathbf{D}^{T}\left(\sum_{i \in \mathcal{R}} \mathbf{f}_{i}^{H} S_{x, i} \mathbf{f}_{i}\right) \mathbf{D H}, \\
\mathbf{B}=\mathbf{H}_{\text {win }}^{T} \mathbf{H}_{\text {win }},
\end{gathered}
$$

where $\mathbf{H}$ is the channel convolution matrix, $\mathbf{H}_{\text {win }}$ is defined in (5), $\mathbf{f}_{i}$ is the $i$ th row of the $N \times N$ DFT matrix, and $\mathbf{D}$ is a diagonal matrix where the diagonal is defined as

$$
g_{k}= \begin{cases}0, & \Delta+1 \leq k \leq \Delta+v+1 \\ 1, & \text { otherwise }\end{cases}
$$

Compared to the MSSNR method, the min-ISI method holds the energy inside the window of size $(\nu+1)$ constant while minimizing a frequency-weighted form of the energy outside the window. The frequency weighting is based on the signal energy at a given frequency bin which can be thought as the ISI energy. The weighting can also be chosen to take channel noise into account by replacing $S_{x, i}$ in (11) with $S_{x, i} / S_{n, i}$ where $S_{n, i}$ is the noise power in subchannel $i$. This weighting function emphasizes the placement of ISI in the frequencies with high SNR (low noise power). A small amount of ISI power in subchannels with low noise power can reduce the overall SNR dramatically. In subchannels with low SNR, however, the noise power is large enough to dominate the ISI power such that the effect of ISI power on the SNR is negligible.

\subsection{The minimum delay spread method}

Schur and Speidel [8] propose another approach to shorten a channel impulse response which can be described as variation of the MSSNR method. The idea behind this approach is to minimize the square of the delay spread of the effective channel impulse response which is defined as

$$
D=\sqrt{\frac{1}{E} \sum_{n=0}^{L_{c}}(n-\tilde{n})^{2}|c[n]|^{2}}
$$

where $\mathbf{c}$ is the effective channel impulse response defined as $\mathbf{c}=$ Hw. $L_{c}$ is the length of the effective channel impulse response, $E=\mathbf{c}^{T} \mathbf{c}$ is the total energy in the effective channel impulse response, and $\tilde{n}$ is the predefined "center of mass."

If we can think of the MSSNR method as weighting the samples of the effective channel impulse response with zero inside the window of size $v+1$ and one elsewhere, the MDS method, on the other hand, weights all samples with the square distance from the "center of mass" which has a similar function to the $\Delta$ delay parameter in the MSSNR or min-ISI methods. The objective function of this method is the square delay spread which can be written as a Rayleigh quotient with $\mathbf{A}$ and $\mathbf{B}$ defined as

$$
\begin{aligned}
& \mathbf{A}=\mathbf{H}^{T} \mathbf{Q} \mathbf{H}, \\
& \mathbf{B}=\mathbf{H}^{T} \mathbf{H},
\end{aligned}
$$

where $\mathbf{Q}$ is a diagonal matrix with the diagonal made of the vector $\left[(0-\tilde{n})^{2},(1-\tilde{n})^{2}, \ldots,\left(L_{w}+L_{h}-1-\tilde{n}\right)^{2}\right]$, and $\tilde{n}$ is the "center of mass."

\section{DIVIDE-AND-CONQUER METHODS}

Each method in the previous section requires a Rayleigh quotient to be optimized. The solution to this optimization problem is a generalized eigenvector of the two matrices. Computing the generalized eigenvectors is a computationally challenging task that requires a heavy computational burden and careful scaling to prevent singularities in the matrix computations. In this section, we propose two suboptimal methods called the divide-and-conquer Rayleighquotient (DC-Rayleigh) and divide-and-conquer eigenvector (DC-eigenvector) methods that can be used with most objective functions that can be written as a Rayleigh quotient. The proposed DC methods divide the calculation of a $N_{w^{-}}$ tap TEQ into smaller problems of finding two-tap TEQs, one per iteration. A unit-tap constraint is placed on each two-tap TEQ. The proposed methods are computationally efficient and do not require any advanced matrix computation that could cause singularity problems.

\subsection{Divide-and-conquer Rayleigh quotient method}

The goal is to optimize an objective function of the form

$$
J=\frac{\mathbf{w}^{T} \mathbf{A} \mathbf{w}}{\mathbf{w}^{T} \mathbf{B w}} .
$$

At the $i$ th iteration, $\mathbf{w}_{i}$ is a $2 \times 1$ vector (a two-tap equalizer), and $\mathbf{A}_{i}$ and $\mathbf{B}_{i}$ are $2 \times 2$ matrices. Assuming a unit-tap constraint on each $\mathbf{w}_{i}$ :

$$
\mathbf{w}_{i}=\left[1, g_{i}\right]^{T}
$$

the objective function becomes

$$
\begin{aligned}
J_{i} & =\frac{\mathbf{w}_{i}^{T} \mathbf{A}_{i} \mathbf{w}_{i}}{\mathbf{w}_{i}^{T} \mathbf{B}_{i} \mathbf{w}_{i}}=\frac{\left[\begin{array}{ll}
1 & g_{i}
\end{array}\right]\left[\begin{array}{ll}
a_{1, i} & a_{2, i} \\
a_{2, i} & a_{3, i}
\end{array}\right]\left[\begin{array}{c}
1 \\
g_{i}
\end{array}\right]}{\left[\begin{array}{ll}
1 & g_{i}
\end{array}\right]\left[\begin{array}{ll}
b_{1, i} & b_{2, i} \\
b_{2, i} & b_{3, i}
\end{array}\right]\left[\begin{array}{c}
1 \\
g_{i}
\end{array}\right]} \\
= & \frac{a_{1, i}+2 a_{2, i} g_{i}+a_{3, i} g_{i}^{2}}{b_{1, i}+2 b_{2, i} g_{i}+b_{3, i} g_{i}^{2}} .
\end{aligned}
$$


TABLE 1: DC-Rayleigh algorithm steps and complexity analysis for MSSNR, min-ISI, and MDS TEQ design, only step 3.1 differs among the TEQ methods.

\begin{tabular}{|c|c|c|c|c|c|}
\hline Step & Description & Multiplications & Additions & Divisions & Square root \\
\hline 1 & Initialize $\mathbf{w}_{\mathrm{TEQ}}=[1]$ & - & - & - & - \\
\hline 2 & Initialize $\mathbf{h}_{0}=\mathbf{h}$ & - & - & - & - \\
\hline 3 & Repeat for $i=1, \ldots, N_{w}-1$ & - & - & - & - \\
\hline 3.1 & MSSNR $\mathbf{A}_{i}(28)$ and $\mathbf{B}_{i}(29)$ & $2\left(L_{h}+i+1\right)$ & $2\left(L_{h}+i\right)$ & - & - \\
\hline 3.1 & $\min -$ ISI $\mathbf{A}_{i}(30)$ and $\mathbf{B}_{i}(29)$ & $2\left(L_{h}+i+N+2\right)$ & $2\left(L_{h}+i+N+1\right)$ & - & - \\
\hline 3.1 & MDS $\mathbf{A}_{i}$ (32) and $\mathbf{B}_{i}$ (33) & $5\left(L_{h}+i\right)+4$ & $5\left(L_{h}+i\right)$ & - & - \\
\hline 3.2 & $g_{i, 1}$ and $g_{i, 2}$ from (19) & 14 & 8 & 2 & 1 \\
\hline 3.3 & $J$ in (25) for $g_{i, 1}$ and $g_{i, 2}$ & 12 & 8 & 2 & - \\
\hline 3.4 & $\mathbf{w}_{\mathrm{TEQ}}=\mathbf{w}_{\mathrm{TEQ}} * \mathbf{w}_{i}$ & $(i-1)$ & $(i-1)$ & - & - \\
\hline 3.5 & $\mathbf{h}_{i}=\mathbf{h}_{i-1} * \mathbf{w}_{i-1}$ & $\left(L_{h}+i\right)$ & $\left(L_{h}+i\right)$ & - & - \\
\hline
\end{tabular}

The assumption that matrix $\mathbf{B}$ is positive definite prevents the denominator in (17) from going to zero for any value of $g_{i}$. Inspection of the $\mathbf{B}$ matrices in the three objective functions in the previous section will show that all are symmetric and positive definite by definition.

Differentiating $J_{i}$ in (17) with respect to $g_{i}$, setting the derivative to zero, and simplifying the result leads to

$$
\begin{gathered}
\left(a_{3, i} b_{2, i}-a_{2, i} b_{3, i}\right) g_{i}^{2}+\left(a_{3, i} b_{1, i}-a_{1, i} b_{3, i}\right) g_{i} \\
+\left(a_{2, i} b_{1, i}-a_{1, i} b_{2, i}\right)=0 .
\end{gathered}
$$

The solutions to the quadratic function of $g_{i}$ in (18) are

$$
g_{i,(1,2)}=\frac{-\left(a_{3, i} b_{1, i}-a_{1, i} b_{3, i}\right) \pm \gamma}{2\left(a_{3, i} b_{2, i}-a_{2, i} b_{3, i}\right)}
$$

where $\gamma$ is

$$
\sqrt{\left(a_{3, i} b_{1, i}-a_{1, i} b_{3, i}\right)^{2}-4\left(a_{3, i} b_{2, i}-a_{2, i} b_{3, i}\right)\left(a_{2, i} b_{1, i}-a_{1, i} b_{2, i}\right)} .
$$

We choose the value of $g_{i}$ among $\left\{g_{i, 1}, g_{i, 2}\right\}$ in (19) that gives the optimal value for $J_{i}$. Once the value for $g_{i}$ is chosen, we have a two-tap TEQ $\mathbf{w}_{i}$ that maximizes the given objective.

Our goal is to maximize the objective for a $N_{w}$-tap TEQ. After the first iteration, we convolve the calculated two-tap TEQ with the channel impulse response $\mathbf{h}$ to obtain an intermediate effective channel impulse response $\mathbf{h}_{1}$. Assuming that this newly calculated intermediate effective channel impulse response is our new channel we repeat the above procedure and calculate a new two-tap TEQ and a new intermediate channel impulse response. This process is repeated $N_{w}-1$ times so that we have $g_{i}$, and hence $\mathbf{w}_{i}$ for $i=1, \ldots, N_{w}-1$. The $N_{w}$-tap TEQ can than be obtained by convolving all twotap TEQs together:

$$
w(k)=w_{1}(k) * w_{2}(k) * \cdots * w_{N_{w}-1}(k),
$$

where $w_{i}(k)$ is the two-tap TEQ obtained at the ith iteration. Table 1 summarizes the steps of the DC-Rayleigh method.

We can also design a two-tap equalizer with a unit-norm constraint (UNC) as

$$
\mathbf{w}_{i}=\left[\sin \theta_{i}, \cos \theta_{i}\right]^{T}
$$

By factoring out $\sin \theta_{i}$, we can rewrite (22) to obtain

$$
\mathbf{w}_{i}=\left[\sin \theta_{i}, \cos \theta_{i}\right]^{T}=\sin \theta_{i}\left[1, \frac{\cos \theta_{i}}{\sin \theta_{i}}\right]^{T}=\sin \theta_{i}\left[1, \eta_{i}\right]^{T}
$$

If we substitute (23) into (17), then the $\sin \theta_{i}$ term would cancel out, which would give the same result as (19).

\subsection{Divide-and-conquer eigenvector method}

The DC-Rayleigh method finds a suboptimal solution of an objective function described as a Rayleigh quotient. In many cases, however, the denominator term of the objective function is constrained to prevent the trivial all-zero TEQ solution. For example in the MSSNR and min-ISI methods the denominator term is to constrain the energy inside the window of length $v+1$. In the MDS method the denominator is constraining the total energy in the effective channel impulse response. The DC-Rayleigh method already places a unit-tap constraint on each two-tap TEQ, which prevents the trivial solution.

The DC-eigenvector method is developed to drop the denominator term from the objective function and optimize the numerator only in order to prevent over-constraining the solution space. The problem is reduced to optimizing the quadratic objective function

$$
J_{i}=\mathbf{w}_{i}^{T} \mathbf{A}_{i} \mathbf{w}_{i}
$$

We apply the same idea in optimizing this objective function by defining a two-tap TEQ as in (16) and rewriting the 
objective function at the ith iteration as

$$
\begin{aligned}
J_{i} & =\mathbf{w}_{i}^{T} \mathbf{A}_{i} \mathbf{w}_{i}=\left[\begin{array}{ll}
1 & g_{i}
\end{array}\right]\left[\begin{array}{ll}
a_{1, i} & a_{2, i} \\
a_{2, i} & a_{3, i}
\end{array}\right]\left[\begin{array}{c}
1 \\
g_{i}
\end{array}\right] \\
& =a_{1, i}+2 a_{2, i} g_{i}+a_{3, i} g_{i}^{2} .
\end{aligned}
$$

Differentiating $J_{i}$ in (25) with respect to $g_{i}$ and setting the derivative to zero gives

$$
g_{i}=-\frac{a_{2, i}}{a_{3, i}} .
$$

Once again we obtain the optimal solution for a two-tap TEQ. Repeating this process $N_{w}-1$ times and convolving the resulting two-tap TEQs together, we obtain the $N_{w}$-tap TEQ.

Note that the DC-Rayleigh method requires the calculation of all entries of both $\mathbf{A}$ and $\mathbf{B}$ matrices at every iteration, but the DC-eigenvector method only requires two entries of the A matrix to be computed in every iteration. The DCeigenvector method also does not require a square root operation, which further reduces the computational complexity and is more suitable for real-time implementation on a programmable digital signal processor.

Similar to the DC-Rayleigh method we can derive the unit-norm constrained DC-eigenvector by replacing $\mathbf{w}_{i}$ in (25) by (23) to obtain

$$
\begin{aligned}
J_{i, \mathrm{UNC}} & =\mathbf{w}_{i}^{T} \mathbf{A}_{i} \mathbf{w}_{i} \\
& =\sin \theta_{i}\left[\begin{array}{ll}
1 & \eta_{i}
\end{array}\right]\left[\begin{array}{ll}
a_{1, i} & a_{2, i} \\
a_{2, i} & a_{3, i}
\end{array}\right] \sin \theta_{i}\left[\begin{array}{c}
1 \\
\eta_{i}
\end{array}\right] \\
& =\sin ^{2} \theta_{i}\left(a_{1, i}+2 a_{2, i} \eta_{i}+a_{3, i} \eta_{i}^{2}\right)
\end{aligned}
$$

which will make $\eta_{i}$ equal to $g_{i}$ in (26) after we solve for $\eta_{i}$. Therefore, both unit-tap constraint and unit-norm constraint in DC-Rayleigh and DC-eigenvector methods should yield the same performance.

\section{APPLICATION OF DIVIDE-AND-CONQUER METHODS}

This section gives detailed derivations on how the MSSNR, min-ISI, and MDS objective functions can be used in conjunction with DC-Rayleigh and DC-eigenvector methods.

Tables 1 and 2 describe the steps and quantify the computations per iteration for the DC-Rayleigh and DCeigenvector methods, respectively. Note that only the calculation of the $\mathbf{A}_{i}$ and $\mathbf{B}_{i}$ matrices differ between methods.

The delay parameter ( $\Delta$ in the min-ISI and MSSNR methods or $\tilde{n}$ in the MDS method) is still an important parameter in the DC methods although it does not appear in the derivation of the DC methods themselves. This parameter is embedded in the $\mathbf{A}_{i}$ and $\mathbf{B}_{i}$ matrices, as it was in the original methods. In [2], a range of 15-35 for the delay parameter caused a change in achieved bitrate of less than $\pm 1 \%$ for MDS, $\pm 2 \%$ for MSSNR, and $\pm 5 \%$ for min-ISI methods. A reasonable initial guess for the delay parameter is the cyclic prefix length $v$ (i.e., 32 for downstream ADSL). When using the DC methods, a delay search could be performed during the first iteration.

\subsection{Application to MSSNR}

In the case of MSSNR, the $\mathbf{A}_{i}$ and $\mathbf{B}_{i}$ matrices used at iteration $i$ are written as

$$
\begin{aligned}
\mathbf{A}_{i} & =\mathbf{H}_{i, \text { win }}^{T} \mathbf{H}_{i, \text { win }}=\left[\begin{array}{ll}
a_{1, i} & a_{2, i} \\
a_{2, i} & a_{3, i}
\end{array}\right] \\
& =\left[\begin{array}{cc}
\sum_{k=\Delta+1}^{\Delta+v+1} h_{i}^{2}(k) & \sum_{k=\Delta+1}^{\Delta+v+1} h_{i}(k) h_{i}(k-1) \\
\sum_{k=\Delta+1}^{\Delta+\nu+1} h_{i}(k) h_{i}(k-1) & \sum_{k=\Delta+1}^{\Delta+\nu+1} h_{i}^{2}(k-1)
\end{array}\right], \\
\mathbf{B}_{i} & =\mathbf{H}_{i, \text { wall }}^{T} \mathbf{H}_{i, \text { wall }}=\left[\begin{array}{ll}
b_{1, i} & b_{2, i} \\
b_{2, i} & b_{3, i}
\end{array}\right] \\
& =\left[\begin{array}{cc}
\sum_{k \in S} h_{i}^{2}(k) & \sum_{k \in S} h_{i}(k) h_{i}(k-1) \\
\sum_{k \in S} h_{i}(k) h_{i}(k-1) & \sum_{k \in S} h_{i}^{2}(k-1)
\end{array}\right],
\end{aligned}
$$

where $h_{i}(k)=h_{i-1}(k) * w_{i}(k)$ for $i=1, \ldots, N_{w-1}, k=$ $0, \ldots, L_{h}+i-1$, and $h_{0}(k)$ is the original channel impulse response. The convolution to obtain the new intermediate channel impulse response is simplified by the fact the first tap of the two-tap TEQ is always set to one; hence, only one multiplication and one addition is required to calculate each tap of the new intermediate impulse response. Also note that $a_{3, i}$ and $b_{3, i}$ are closely related to $a_{1, i}$ and $b_{1, i}$, respectively, in that they differ in only two elements of the sums hence can be derived from each other without recomputing the square of the sums.

\subsection{Application to min-ISI}

In the case of min-ISI, the $\mathbf{B}_{i}$ matrix is the same as given in (29) and the elements of $\mathbf{A}_{i}$ are defined as

$$
\begin{gathered}
a_{1, i}=\sum_{k \in \mathcal{R}}\left[\sum_{n=0}^{N-1} h_{i}(n) s(k-n)\right]^{2}, \\
a_{2, i}=\sum_{k \in \mathcal{R}}\left[\sum_{n=0}^{N-1} h_{i}(n) s(k-n) \sum_{n=0}^{N-1} h_{i}(n) s(k-1-n)\right]^{2}, \\
a_{3, i}=\sum_{k \in \mathcal{R}}\left[\sum_{n=0}^{N-1} h_{i}(n) s(k-1-n)\right]^{2},
\end{gathered}
$$

where $\delta=\{1, \ldots, \Delta, \Delta+v+2, \ldots, N\}$ and $s(n)$ is the timedomain equivalent of the frequency-domain weighting function $S_{x, i}$ and is defined as

$$
s(n)=\sum_{i=0}^{N-1} S_{x, i} e^{j(2 \pi / N) i n} .
$$

The application of DC methods to the min-ISI objective function requires first the calculation of the time-domain weighting function $s(n)$, which can be performed with an $N$-point inverse FFT. This calculation needs to be done only 
TABLE 2: Implementation complexity of DC-eigenvector algorithms for MSSNR, min-ISI, and MDS TEQ design methods. Only step 3.1 differs among the TEQ methods.

\begin{tabular}{|c|c|c|c|c|c|}
\hline Step & Description & Multiplications & Additions & Divisions & Square root \\
\hline 1 & Initialize $\mathbf{w}_{\mathrm{TEQ}}=[1]$ & - & - & - & - \\
\hline 2 & Initialize $\mathbf{h}_{0}=\mathbf{h}$ & - & - & - & - \\
\hline 3 & Fix $\Delta$. For $i=1, \ldots, N_{w}-1$ & - & - & - & - \\
\hline 3.1 & MSSNR $\mathbf{A}_{i}$ (28) & $\left(L_{h}+i-v\right)$ & $\left(L_{h}+i-v-1\right)$ & - & - \\
\hline 3.1 & $\min -$ ISI $\mathbf{A}_{i}(30)$ & $\left(L_{h}+i-v\right)+2(N+1)$ & $\left(L_{h}+i-v-1\right)+2(N+1)$ & - & - \\
\hline 3.1 & $\operatorname{MDS} \mathbf{A}_{i}$ (32) & $3\left(L_{h}+i\right)+2$ & $3\left(L_{h}+i\right)$ & - & - \\
\hline 3.2 & $g(26)$ & - & - & 1 & - \\
\hline 3.3 & $\mathbf{w}_{\mathrm{TEQ}}=\mathbf{w}_{\mathrm{TEQ}} * \mathbf{w}_{i}$ & $(i-1)$ & $(i-1)$ & - & - \\
\hline 3.4 & $\mathbf{h}_{i}=\mathbf{h}_{i-1} * \mathbf{w}_{i-1}$ & $\left(L_{h}+i\right)$ & $\left(L_{h}+i\right)$ & - & - \\
\hline
\end{tabular}

once and not for every iteration. However the inner sums in (30) are required for every iteration which adds to the computational complexity compared to the MSSNR objective function. As a side benefit, the DC methods get around a restriction of the min-ISI method that the TEQ length could exceed the CP length by designing two taps at a time.

\subsection{Application to MDS}

For the MDS objective functions, the $\mathbf{A}_{i}$ and $\mathbf{B}_{i}$ matrix elements are defined as

$$
\begin{gathered}
a_{1, i}=\sum_{k=0}^{L_{c}}(k-\tilde{n})^{2} h_{i}^{2}(k), \\
a_{2, i}=\sum_{k=0}^{L_{c}}(k-\tilde{n})(k-1-\tilde{n}) h_{i}(k) h_{i}(k-1), \\
a_{3, i}=\sum_{k=0}^{L_{c}}(k-1)(k-1-\tilde{n})^{2} h_{i}^{2}, \\
b_{1, i}=\sum_{k=0}^{L_{c}} h_{i}^{2}(k), \\
b_{2, i}=\sum_{k=0}^{L_{c}} h_{i}(k) h_{i}(k-1), \\
b_{3, i}=\sum_{k=0}^{L_{c}} h_{i}^{2}(k-1) .
\end{gathered}
$$

As with the MSSNR method the calculation of both $a_{3, i}$ and $b_{3, i}$ can be based on $a_{1, i}$ and $b_{1, i}$, respectively, to avoid recalculating the sum of squares. Even with these savings, however, the MDS method requires all sums to be over the entire length of the intermediate channel impulse response doubling the computational complexity compared to the former two methods.

\subsection{Comparison of computational complexity}

We compare the computation complexity of the application of both the DC-Rayleigh and DC-eigenvector methods to all three objective functions in this section. For a fair comparison of computational complexity, we replace the eigenvalue decomposition in the original methods by the iterative power method [13] since only the dominant eigenvalue and its corresponding eigenvector are needed. We assume 10 iterations for the power method as in [14].

Table 3 summarizes the original TEQ design methods and their computational costs, whereas Tables 1 and 2 summarize the DC-Rayleigh and DC-eigenvector methods and their computational complexity, respectively.

Both proposed methods have reduced computational complexity when compared to the original methods and are better suited for real-time implementation on digital signal processors because they avoid any matrix calculations that require careful scaling. The complexity gap between the original and proposed methods increases with increasing $N_{w}$ because the dominant cost savings are from the matrix operations performed on $N_{w} \times N_{w}$ matrices in the original methods.

Table 4 lists the computational complexity for each of the methods for a moderate length TEQ of size $N_{w}=16$ and $N=L_{h}=512$, and $v=32$, by assuming 10 iterations in the power method for the original methods. The largest complexity reduction is $24 \%$ and $15 \%$ for the MSSNR objective function for the DC-Rayleigh and DC-eigenvector methods, respectively. Percentage savings in all cases would increase for longer TEQs.

\section{SIMULATION RESULTS}

We showed in the previous section that the divide-andconquer methods, especially the DC-eigenvector method, have significant complexity savings over the original methods. In this section, we present simulation results to analyze the bitrate performance of the proposed methods. It is worth noting that the DC-Rayleigh method communication performance is bounded above by the performance of the original method used because it optimizes the same function but two taps at a time. Since we fix the previous taps at every iteration, we are not guaranteed to obtain the optimal solution. It is not possible to determine the upper-bound performance for the DC-eigenvector method in terms of the original methods since the DC-eigenvector method uses different constraints compared to the original methods. 
TABLE 3: Implementation complexity of the MSSNR, min-ISI, and MDS methods.

\begin{tabular}{|c|c|c|c|c|}
\hline Step & Description & Multiplications & Additions & Divisions \\
\hline 1 & A and $\mathbf{B}$ for MSSNR (8) & $N_{w}\left(L_{h}+2 N_{w}-2\right)$ & $N_{w}\left(L_{h}+2 N_{w}-3\right)$ & - \\
\hline 1 & $\mathbf{A}$ and $\mathbf{B}$ for min-ISI (11) & $N_{w}\left(L_{h}+2 N_{w}-1+N\right)$ & $N_{w}\left(L_{h}+2 N_{w}-2+N\right)$ & - \\
\hline 1 & A and $\mathbf{B}$ for MDS (14) & $2 N_{w}\left(L_{h}+2 N_{w}-2\right)+L_{h}+N_{w}-1$ & $2 N_{w}\left(L_{h}+2 N_{w}-3\right)+L_{h}+N_{w}-1$ & - \\
\hline 2 & Cholesky Decomposition B & $N_{w}^{3}$ & $N_{w}^{3}$ & - \\
\hline 3 & $(\sqrt{\mathbf{B}})^{-1}[11]$ & $\left(5 N_{w}^{3}+N_{w}\right) / 3$ & $\left(5 N_{w}^{3}+N_{w}\right) / 3$ & - \\
\hline 4 & $\mathbf{c}=(\sqrt{\mathbf{B}})^{-1} \mathbf{A}\left(\sqrt{\mathbf{B}^{T}}\right)^{-1}$ & $2 N_{w}^{3}$ & $2 N_{w}^{2}\left(N_{w}-1\right)$ & - \\
\hline 6 & \multicolumn{4}{|c|}{ Power method to find eigenvector corresponding to the minimum eigenvalue of $\mathbf{C}$} \\
\hline 6.1 & Calculate $\mathbf{C}^{-1}[11]$ & $\left(5 N_{w}^{3}+N_{w}\right) / 3$ & $\left(5 N_{w}^{3}+N_{w}\right) / 3$ & 0 \\
\hline 6.2 & Initialize $\mathbf{l}^{(0)}$ & - & - & - \\
\hline 6.3 & $\mathbf{z}^{(k)}=\mathbf{C}^{-1} \mathbf{l}^{(k-1)}$ & $N_{w}^{2}$ & $\left(N_{w}-1\right) N_{w}$ & 0 \\
\hline 6.4 & $\mathbf{l}_{\mathrm{opt}}^{(k)}=\mathbf{z}^{(k)} /\left\|\mathbf{z}^{(k)}\right\|$ & $N_{w}$ & $N_{w}-1$ & $N_{w}$ \\
\hline 6.5 & $\lambda^{(k)}=\left[\mathbf{l}^{(k)}\right]^{T} \mathbf{C}^{-1} \mathbf{l}^{(k)}$ & $\left(N_{w}+1\right) N_{w}$ & $N_{w}^{2}-1$ & 0 \\
\hline 6.6 & if $\left|\lambda^{(k)}-\lambda^{(k-1)}\right|>$ threshold, & step 6.3 & - & - \\
\hline 7 & $\mathbf{w}_{\mathrm{opt}}=\left(\sqrt{\mathbf{B}^{T}}\right)^{-1} \mathbf{l}_{\mathrm{opt}}^{(k)}$ & $N_{w}^{2}$ & $\left(N_{w}-1\right) N_{w}$ & 0 \\
\hline
\end{tabular}

TABle 4: Tradeoff between bitrate performance and complexity (multiplications) of the original and the two divide-and-conquer variations of each TEQ design method for $v=32, N_{w}=16$, and $L_{h}=512$. Complexity of original methods assume that the power method is run for 10 iterations.

\begin{tabular}{lcrrr}
\hline Method & $\begin{array}{c}\text { Bitrate } \\
(\mathrm{Mbps})\end{array}$ & Complexity & Bitrate & Complexity \\
\hline MSSNR original & 7.96 & 101808 & $100 \%$ & $100 \%$ \\
MSSNR Rayleigh & 7.34 & 23925 & $92 \%$ & $24 \%$ \\
MSSNR eigenvector & 7.74 & 15225 & $97 \%$ & $15 \%$ \\
\hline Min-ISI original & 8.02 & 110016 & $100 \%$ & $100 \%$ \\
Min-ISI Rayleigh & 7.40 & 39315 & $92 \%$ & $36 \%$ \\
Min-ISI eigenvector & 7.70 & 30615 & $96 \%$ & $28 \%$ \\
\hline MDS original & 7.72 & 111007 & $100 \%$ & $100 \%$ \\
MDS Rayleigh & 7.45 & 47355 & $96 \%$ & $43 \%$ \\
MDS eigenvector & 7.58 & 31335 & $98 \%$ & $28 \%$ \\
\hline
\end{tabular}

All simulations are based on the commonly used eight carrier-serving-area (CSA) loops that were obtained from the UT Austin Matlab DMTTEQ Toolbox [15]. The CSA loops are placed in cascade with two fifth-order high-pass Chebyshev filters. The first filter has a turn-on frequency of $4.8 \mathrm{kHz}$ and simulates the effect of the splitter that separates the voice-band from the data-band. The second filter is used to separate the upstream from the downstream in frequency division multiplexing at a frequency of $138 \mathrm{kHz}$.

A transmit signal power of $26 \mathrm{dBm}$ on a $100 \Omega$ load is assumed. The thermal noise is modeled as white Gaussian noise with $-140 \mathrm{dBm} / \mathrm{Hz}$ spectral power. Near-end crosstalk noise is introduced for 8 ISDN disturbers as described in the ADSL specifications [1]. All methods make use of an ideal estimate of the channel impulse response.

The delay parameter is chosen based on a heuristic search that gives satisfactory performance with minimal complexity [14]. The method uses a rectangular window of size $v+1$ that is slid over the original channel impulse response so that the SSNR as defined in (4) is maximized. The index of the first nonzero sample of the window is chosen as the delay parameter.

Simulations are carried out for 300 DMT symbols carrying quadrature phase-shift keying (QPSK) signals in all subchannels, except for subchannel 0 (voiceband) which is not used for data, subchannels 1-5 (ISDN band) which are not used for data, and subchannel N/2 + 1 which cannot carry complex symbols. At the receiver, an FIR TEQ filters the received noisy signal, and passes its output through the cyclic prefix removal block and the FFT. A one-tap FEQ per subchannel rotates the symbols in each subchannel. (We designed the FEQ tap on each subchannel to be optimal in a mean-squared error sense.) The rotated symbols are then compared to the transmitted symbols in each subchannel, and the difference is the error signal, from which the receiver SNR is calculated. Based on the SNR in each subchannel, we calculate the total bitrate achievable for the given TEQ by using

$$
b_{\mathrm{DMT}}=\sum_{k \in \mathcal{R}}\left\lfloor\log _{2}\left(1+\frac{\mathrm{SNR}_{i}}{\Gamma}\right)\right\rfloor,
$$

where $\mathcal{R}$ is a set of indices for all used subchannels, $\mathrm{SNR}_{i}$ is the SNR in subchannel $i$, and the SNR gap $\Gamma$ is $10.8 \mathrm{~dB}$ [16].

Figure 1 shows the impulse response of CSA loop 1 and the shortened or effective impulse response obtained with a 16-tap TEQ designed with the proposed MSSNRDC-Rayleigh method. Figure 2 compares the performance of all nine methods for all 8 CSA loops with the matchedfilter bound (MFB) and the case where no TEQ is used at all. The motivation of using a TEQ is apparent due to the gap in communication performance when no TEQ is used. When comparing the original methods among each other in achieved bitrate, the MSSNR and min-ISI methods perform closely while outperforming the MDS method. All methods seem to perform relatively close to the MFB although other TEQ design methods that get even closer to the MFB 


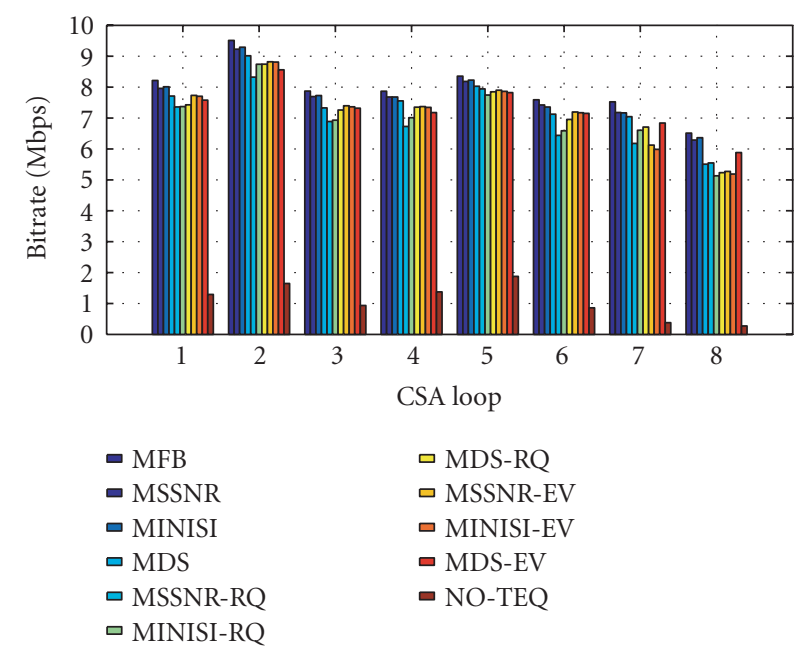

FIgure 2: Performance of all methods on 8 CSA loops with TEQ length $N_{w}=16$, symbol length $N=512$, channel length $L_{h}=512$, and cyclic prefix length $v=32$.

exist [2]. As expected, the proposed suboptimal divide-andconquer methods generally perform worse than the original methods. However, on CSA loops 6 and 8, the proposed MDS-DC-eigenvector method actually outperforms the original MDS method. This could be expected since none of the methods directly optimize bitrates but alternative objective functions such as the delay spread in this case. Since optimizing the delay spread is not equivalent to optimizing the bitrate, one can sometimes expect the bitrate to increase while the delay spread decreases.

Another observation from Figure 2 is that most of the time, the DC-eigenvector method outperforms the DCRayleigh method. At first thought, one might think that the DC-Rayleigh method should perform better because it solves the original objective functions as opposed to a simplified one, as the DC-eigenvector method does. As mentioned in Section 3.2, the DC-Rayleigh method may be overconstraining the solution due to the new constraint on the first tap of the two-tap equalizers designed at every iteration. For all three original methods in this paper, the denominator of the Rayleigh quotient serves mostly as a constraint to prevent the all-zero trivial solution for the TEQ. The divide-andconquer methods already have a unit-tap constraint built into them. Thus, removing the original constraint expands the solution space, and the DC-eigenvector method is able to find better solutions most of the time while having lower implementation complexity. Taking into account the reduction in computational complexity when compared to the DCRayleigh method the DC-eigenvector method seems to be a better choice in general.

The primary motivation of the proposed DC methods is to reduce the computational complexity and avoid matrix computations that require careful scaling in return for some communication performance loss. Figure 3(a) maps all methods referred in this paper onto a two-dimensional space with one axis representing the computational complexity and the other communication performance. The best solutions would lie in the lower right corner of this map where performance is maxima and computational complexity is minimal. This plot is obtained by averaging the bitrate numbers obtained for each method on each of the eight CSA loops. We see from this plot that the MSSNR-DC-eigenvector method is the choice when computational complexity is the major deciding factor. If, however, communication performance is the only factor, then the original min-ISI or the MSSNR methods seemed to be the best choice. Complexity of the original methods assume that the power method is run for 10 iterations. One could easily argue that the performance gap between the proposed DC methods and the original methods is so small (on the order of $0.5 \mathrm{Mbps}$ ) that the extra complexity and implementation hardship due to matrix operations is not justified. This plot also reveals that for all DCRayleigh methods there exists a method that gets better performance with lower computational complexity. A similar argument holds for the min-ISI objective function which seems not to perform as well as the other two objective functions when DC methods are applied. The MSSNR-DC-eigenvector method gives on average better performance with less complexity compared to the min-ISI when DC methods are applied. Figure 3(b) shows performance for all methods under varying numbers of TEQ taps. The graph shows that most methods settle around an upper-bound performance with a 10-tap TEQ. The DC-Rayleigh methods actually reduce bitrate performance with increasing numbers of taps. This again could be explained by the fact that none of these methods directly optimize the bitrate. It turns out that the DC-Rayleigh method tends to use the additional freedom of more TEQ taps in the wrong direction in terms of bitrate performance.

Finally we analyze the effect of channel estimation error on each method. The channel estimation error is modeled as additive white Gaussian noise on the ideal (real) channel impulse response. The noisy channel estimate is used in the calculations of the TEQ coefficients with each method while the performance estimation is done using the real channel impulse response.

As shown in Figure 4 the performance of all methods increases with increasing SNR of the channel estimation error. For SNRs higher than $80 \mathrm{~dB}$ the original methods outperform the iterative methods by similar margin as with an ideal channel so the noise is too low to have an effect on the results. The performance gap between the original and DC methods increases for SNRs lower than $80 \mathrm{~dB}-90 \mathrm{~dB}$. The worstcase additional performance loss of the DC methods over the original methods is around 3\% for the MSSNR and minISI-based DC methods and about 14\% for the MDS-based DC methods. So we can conclude that the MDS method is more sensitive to channel estimation errors when used in conjunction with the proposed DC methods. This conclusion also agrees with the results in [10].

Although the DC-Rayleigh method follows the trend of the original method with drastic performance reductions at lower SNRs, the DC-eigenvector method delivers about 30$40 \%$ of the peak bitrate even with bad channel estimates. This again may be explained by the fact that the DC-eigenvector 


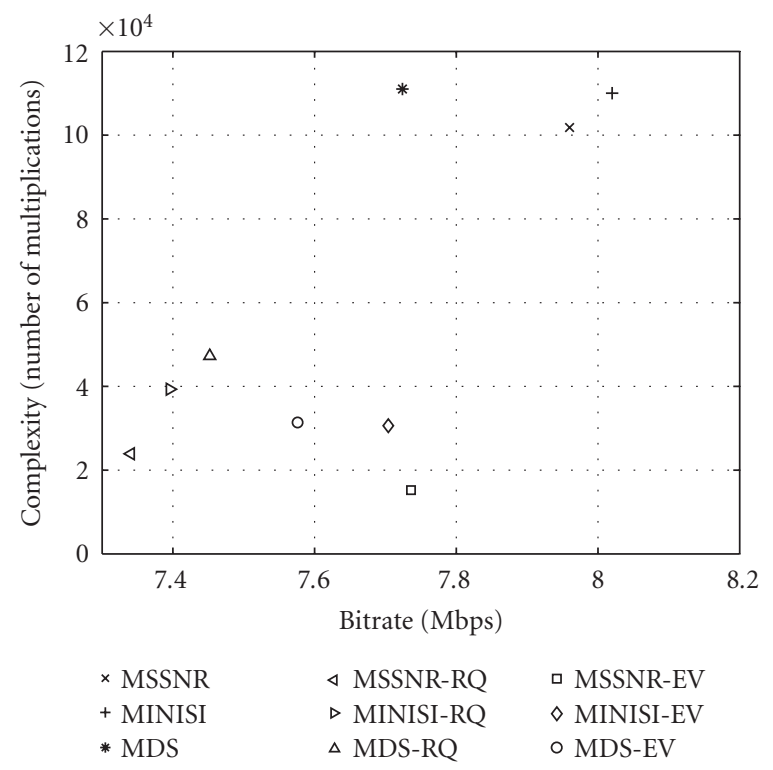

(a)

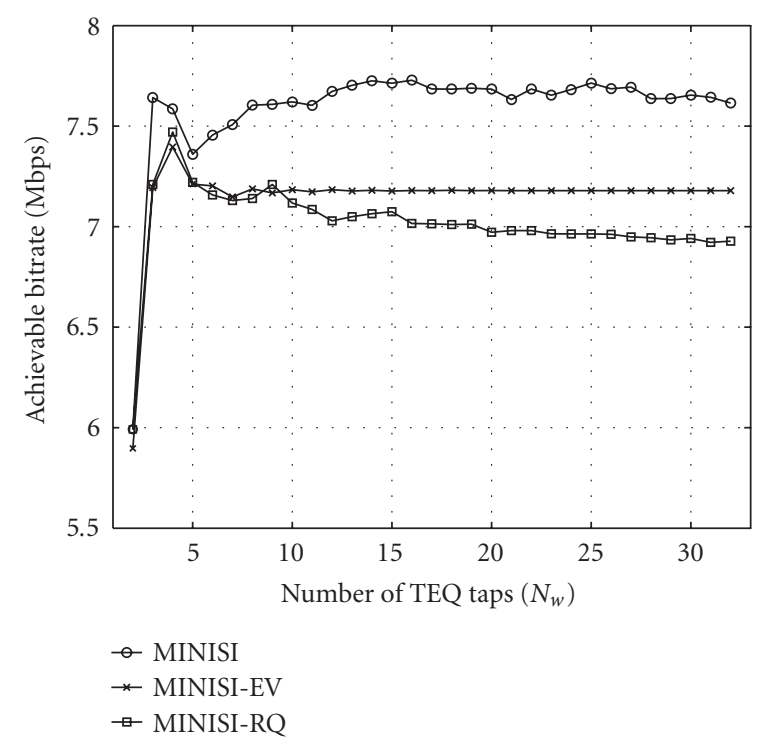

(c)

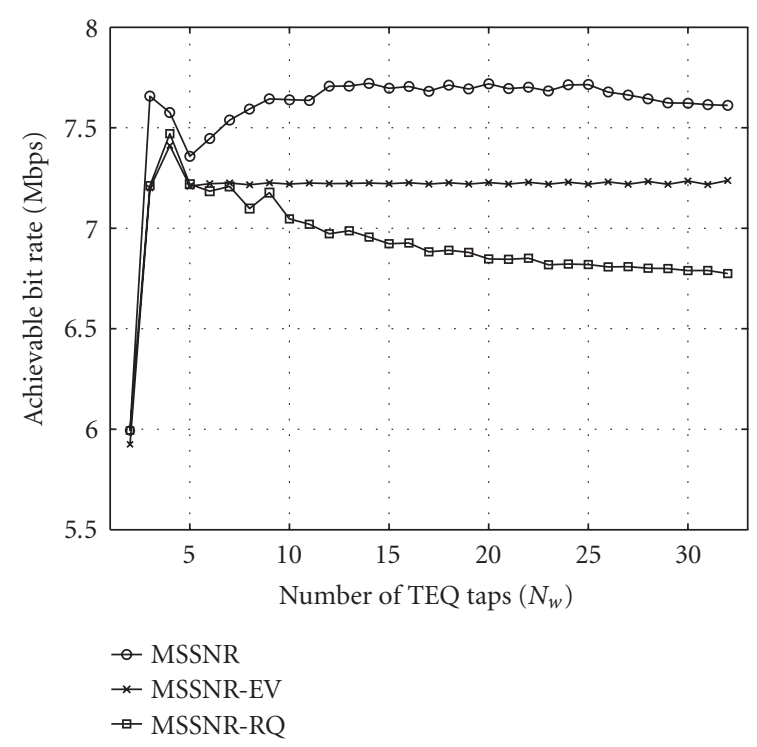

(b)

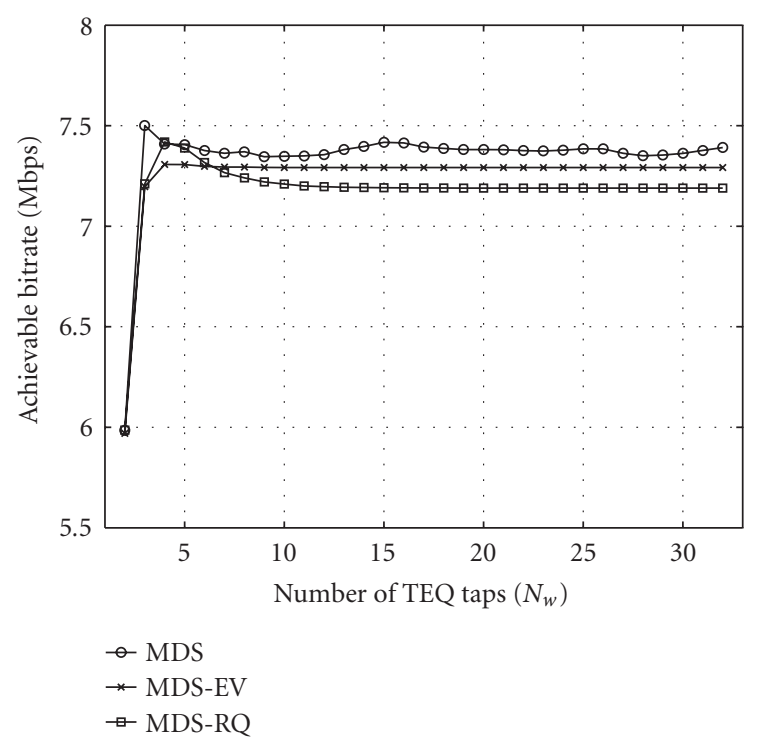

(d)

FIgURE 3: With symbol length $N=512$ and channel length $L_{h}=512$, communication performance versus (a) implementation complexity for all methods with TEQ length $N_{w}=16$ and cyclic prefix length $v=32$, where the bitrates are taken as the average over all eight CSA loops; (b) TEQ length for MSSNR methods; (c) TEQ length for min-ISI methods; and (d) TEQ length for MDS methods. EV means the DC-eigenvalue method and RQ means the DC-Rayleigh method.

methods are less constrained hence have a larger space to find a better solution even with noisy channel estimates. The original methods as well as the DC-Rayleigh methods practically stop working at low SNR situations delivering only about $10 \%$ of the peak bitrate with $20 \mathrm{~dB}$ estimation noise.

\section{CONCLUSION}

The design of a time-domain equalizer (TEQ) for discrete multitone modulation has been studied extensively and a number of methods can deliver bitrates close to the upper bound of achievable performance. Many of these highperformance methods can mathematically be classified as an optimization of a Rayleigh quotient, which requires computationally intensive matrix decompositions to solve directly. The focus of this paper is to reduce the computational complexity by avoiding matrix decompositions. We propose an iterative refinement approach in which the TEQ length starts at two taps and increases by one tap at each iteration. 


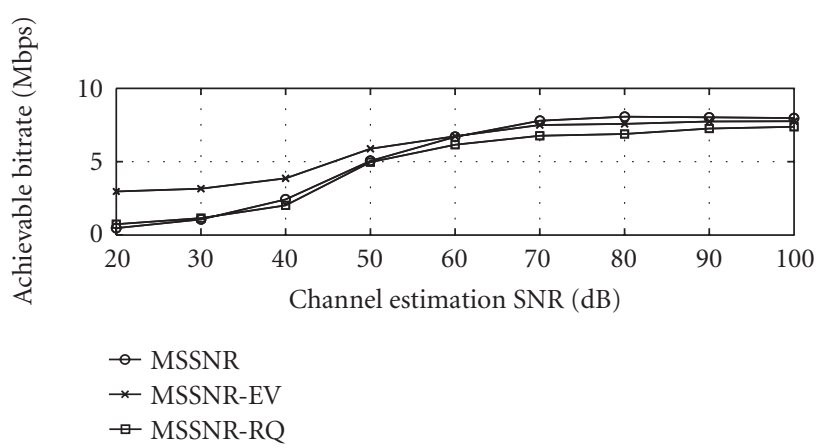

(a)

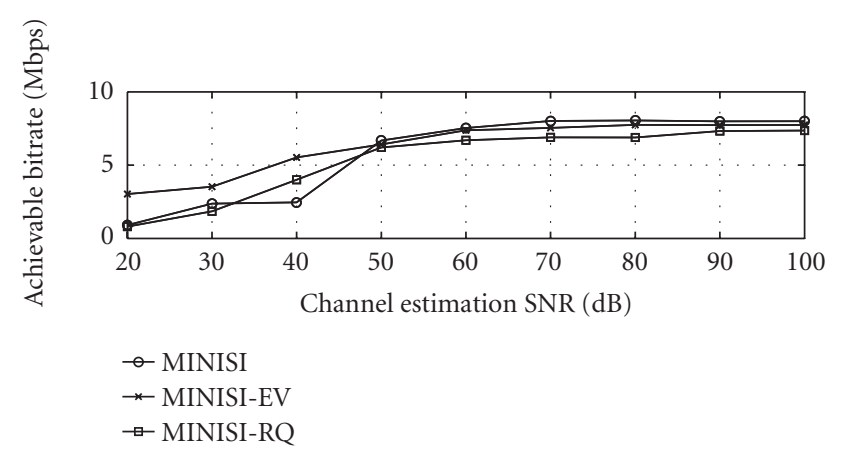

(b)

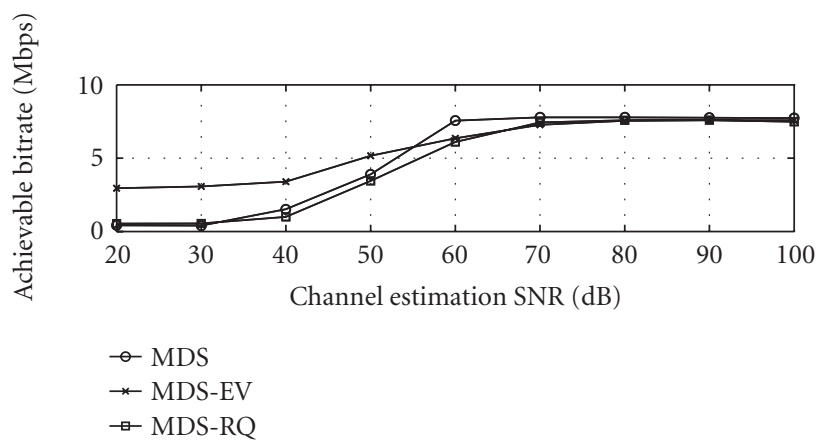

(c)

Figure 4: Performance of all methods on CSA loop 1 with TEQ length $N_{w}=16$, symbol length $N=512$, channel length $L_{h}=512$, cyclic prefix length $v=32$, and SNR of channel estimation error. Estimation error is modeled as additive white Gaussian noise. Performance is averaged over 10 runs.

The first method is the divide-and-conquer Rayleigh quotient (DC-Rayleigh) method. The DC-Rayleigh gives an approximate solution to the Rayleigh quotient optimization problem. Our simulation results show that the proposed DCRayleigh method gives close to ideal performance with reduced computational complexity. The fact that the proposed DC-Rayleigh method introduces an additional unit-tap constraint on the solutions motivates us to further simplify the TEQ design methods by dropping the divisor term from the Rayleigh quotient. This yields to a quadratic cost functions with eigenvector solutions. The second method is the divideand-conquer eigenvector method (DC-eigenvector), which solves the eigenvalue problem approximately with further reduced complexity.

We apply both divide-and-conquer methods to optimize the objective functions of three different TEQ design methods. The methods are the maximum shortening signal-tonoise ratio, minimum intersymbol interference, and minimum delay spread (MDS). Complexity analysis and simulations results show that the proposed methods reduce the computational complexity of the original methods with minor performance degradation. In fact, the proposed iterative refinement approach provides a range of communication performance versus implementation complexity tradeoffs for any TEQ method that fits the Rayleigh quotient framework. The measure of communication performance depends on the objective function used by the TEQ method.

\section{REFERENCES}

[1] "ANSI T1.413-1995, Network and customer installation interfaces: Asymmetrical digital subscriber line (ADSL) metallic interface," printed from: Digital Subscriber Line Technology by T. Starr, J. M. Cioffi, and P. J. Silverman, Prentice-Hall, 1999.

[2] R. K. Martin, K. Vanbleu, M. Ding, et al., "Unification and evaluation of equalization structures and design algorithms for discrete multitone modulation systems," IEEE Transactions Signal Processing, vol. 53, no. 10, part 1, pp. 3880-3894, 2005.

[3] J. S. Chow and J. M. Cioffi, "A cost-effective maximum likelihood receiver for multicarrier systems," in Proceedings of IEEE International Conference on Communications (ICC '92), vol. 2, pp. 948-952, Chicago, Ill, USA, June 1992.

[4] K. Van Acker, G. Leus, M. Moonen, O. van de Wiel, and T. Pollet, "Per tone equalization for DMT-based systems," IEEE Transactions on Communications, vol. 49, no. 1, pp. 109-119, 2001.

[5] M. Ding, Z. Shen, and B. L. Evans, "An achievable performance upper bound for discrete multitone equalization," in Proceedings of IEEE Global Telecommunications Conference (GLOBECOM '04), vol. 4, pp. 2297-2301, Dallas, Tex, USA, November-December 2004.

[6] P. J. W. Melsa, R. C. Younce, and C. E. Rohrs, "Impulse response shortening for discrete multitone transceivers," IEEE Transactions on Communications, vol. 44, no. 12, pp. 1662 1672, 1996. 
[7] G. Arslan, B. L. Evans, and S. Kiaei, "Equalization for discrete multitone transceivers to maximize bit rate," IEEE Transactions Signal Processing, vol. 49, no. 12, pp. 3123-3135, 2001.

[8] R. Schur and J. Speidel, "An efficient equalization method to minimize delay spread in OFDM/DMT systems," in Proceedings of IEEE International Conference on Communications (ICC '01), vol. 5, pp. 1481-1485, Helsinki, Finland, June 2001.

[9] R. K. Martin, K. Vanbleu, M. Ding, et al., "Implementation complexity and communication performance tradeoffs in discrete multitone modulation equalizers," to appear in IEEE Transactions Signal Processing, http://www.ece.utexas.edu/ bevans/papers/2005/equalizationII.

[10] M. Ding, B. L. Evans, and I. Wong, "Effect of channel estimation error on bit rate performance of time domain equalizers," in Proceedings of 38th IEEE Asilomar Conference on Signals, Systems and Computers, vol. 2, pp. 2056-2060, Pacific Grove, Calif, USA, November 2004.

[11] G. H. Golub and C. F. Van Loan, Matrix Computation, John Hopkins University Press, Baltimore, Md, USA, 3rd edition, 1996.

[12] C. Yin and G. Yue, "Optimal impulse response shortening for discrete multitone transceivers," IEE Electronics Letters, vol. 34, no. 1, pp. 35-36, 1998.

[13] J. W. Demmel, Applied Numerical Linear Algebra, SIAM, Philadelphia, Pa, USA, 1997.

[14] B. Lu, L. D. Clark, G. Arslan, and B. L. Evans, "Fast TimeDomain Equalization for Discrete Multitone Modulation Systems," in Proceedings of IEEE Digital Signal Processing Workshop, Hunt, Tex, USA, October 2000.

[15] G. Arslan, M. Ding, B. Lu, M. Milosevic, Z. Shen, and B. L. Evans, "MATLAB DMTTEQ Toolbox 3.1," 2003, Available at: http://www.ece.utexas.edu/ bevans/projects/adsl/dmtteq/ dmtteq.html.

[16] N. Al-Dhahir and J. M. Cioff, "A bandwidth-optimized reduced-complexity equalized multicarrier transceiver," IEEE Transactions on Communications, vol. 45, no. 8, pp. 948-956, 1997.

Güner Arslan received his Ph.D. degree in electrical engineering (2000) at the University of Texas at Austin in Austin, Texas, USA. His dissertation was entitled Equalization for Discrete Multitone Transceivers. He received his M.S. degree in electronics and communications engineering from Yildiz Technical University, Istanbul, Turkey in 1998, and his B.S. degree in electronics and communications engineering as Vale-

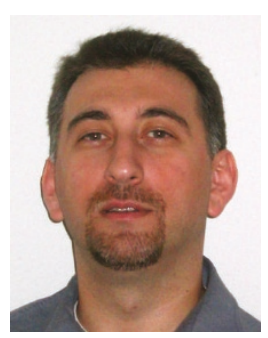
dictorian of his class from Yildiz University, Kocaeli, Turkey in 1994. He is currently a Senior Systems Design Engineer in the wireless product division of Silicon Laboratories based in Austin, Texas, USA. He is also an Adjunct Faculty in the Electrical and Computer Engineering Department at the University of Texas at Austin. His research interests are in digital signal processing, communications systems, and embedded real-time digital signal processing. He is a Member of IEEE Signal Processing and Communication Societies.
Biao Lu received her Ph.D. degree in electrical engineering (2000) and M.S.E.E. degree (1997) from the University of Texas at Austin in Austin, Texas, USA. Her dissertation was entitled Wireline Channel Estimation and Equalization. She received her B.S. degree in biomedical engineering (1992) from the Capital Institute of Medicine in Beijing, China. Since 2000, she has been with Schlumberger in Houston, Texas, USA, where she is currently a Senior Software Engineer in telemetry systems. Her research interests include signal processing, image processing, neural networks, and embedded systems.

Lloyd D. Clark received his B.S., M.S., and Ph.D. degrees in electrical engineering and computer science from the Massachusetts Institute of Technology in 1984, 1986, and 1990, respectively. From 1990 to 2003, he held various positions at the Schlumberger Austin Technology Center in Austin, Texas, USA, including Principal Engineer and Research Scientist. While at Schlumberger, he designed and developed wireline telemetry

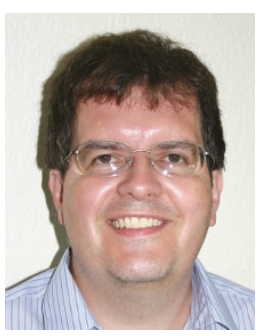
systems for well logging applications for the oil field, as well as wireless metering systems. Since 2004, he has been a Principal Scientist with Ticom Geomatics in Austin, Texas, USA, where he has been the technical lead on several wireless geolocation projects. He holds several patents, has published several technical papers, and has coadvised graduate students at both MIT and the University of Texas at Austin.

Brian L. Evans is Professor of Electrical and Computer Engineering at the University of Texas at Austin in Austin, Texas, USA. His B.S.E.E.C.S. (1987) degree is from the Rose-Hulman Institute of Technology in Terre Haute, Indiana, USA, and his M.S.E.E. (1988) and Ph.D.E.E. (1993) degrees are from the Georgia Institute of Technology in Atlanta, Georgia, USA. From 1993 to 1996, he was a Postdoctoral Researcher at the Uni-

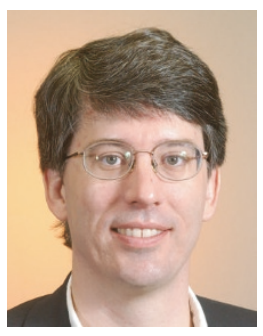
versity of California, Berkeley, in design automation for embedded digital systems. At UT Austin, his research group develops signal quality bounds, optimal algorithms, low-complexity algorithms, and real-time embedded software of high-quality image halftoning for desktop printers, smart image acquisition for digital still cameras, high-bitrate equalizers for multicarrier ADSL receivers, and resource allocation for multiuser OFDM base stations. He is the architect of the Signals and Systems Pack for Mathematica. He received a 1997 US National Science Foundation CAREER Award. 